\title{
Clustering-Based Detection of Anatomical Features on Organic Shapes
}

\author{
Konrad Sickel $^{1}$, Vojtech Bubnik ${ }^{2}$ \\ ${ }^{1}$ Pattern Recognition Lab, Department of Computer Science, \\ Friedrich-Alexander-Universität Erlangen-Nürnberg, Martensstr. 3, 91058 Erlangen \\ ${ }^{2}$ Siemens Hearing Instruments, Inc., 10 Constitution Ave, Piscataway, NJ 08854, USA \\ konrad.sickel@informatik.uni-erlangen.de
}

\begin{abstract}
Robust and accurate automatic detection of anatomical features on organic shapes is a challenging task. Despite a rough similarity, each shape is unique. To cope with this variety, we propose a novel clustering-based feature detection scheme. The scheme can be used as a standalone feature detection scheme or it can provide meaningful starting points for surface analyzing-based detection algorithms. The scheme includes the identification of a representative set of shapes and the usage of a specialized iterative closest point algorithm for the registration of shapes, which is followed by the projection of the features using the transformation matrix of the registration. Evaluation is based on a large set of expert annotated shapes and showed superior performance compared to state-of-the-art surface analyzing methods. Accuracy increased of $32 \%$ and detection of all features is ensured.
\end{abstract}

\section{Introduction}

Shape analysis typically involves the abstraction of complex structures by removing redundant details. It captures the essence of geometry via a representative set of distinctive features, such as 3-D points, planes, curves or areas. These features can be employed for classification, registration and for driving the automation of shape transformations. The problem is very challenging due to the variability of organic surfaces. In this work, we focus on the robust and accurate detection of anatomical features found on ear impressions. An example ear impression with annotated features is given in Fig. 1. Theses features provide the basic structure for the automatic design of customized in-the-ear hearing aids $[1,2]$.

Previous work in this field was reported by Paulsen et al., they employed anatomical features (landmarks) to build a statistical shape model of the human ear canal for shape analysis and the automatic design of customized hearing aids $[2,3]$. Zouhar et al. focused on the detection of anatomical features to guide a fast registration of $3-\mathrm{D}$ ear impressions and also for automation purposes $[4,5]$. Baloch et al. worked on the detection of a canonical ear signature to capture the structure of an ear impression [6]. So far, the proposed algorithms 
for feature detection on ear impressions were solely based on the analysis of surface properties, like peaks, depressions, concavities, ridges and bumps. Hence, the result is sensitive to the quality of the acquired ear impression mesh and can fail in case of unusual ear impressions [6]. Problematic cases are characterized by containing large amounts of excess material, very short ear canals, holes all over the impression and a very jagged opening contour at the bottom of the impression. Furthermore, due to the shape variety some features will not always be very distinctive. We specifically address the named problems by enhancing the current methods with a clustering-based detection (CBD) scheme as described in the following.

\section{Materials and Methods}

Our CBD scheme is based on three methods: (i) robust alignment of ear impressions, (ii) identification of a representative set of ear impressions, and (iii) feature projection from one impression to another.

\subsection{Robust Alignment of Ear Impressions}

Similar to Zouhar et al. [4], we use a specifically adapted version of an iterative closest point (ICP) algorithm to register ear impressions. In contrast to them, we employ a version without previously detected features, since we want to be independent of them. Our approach is divided in two steps: (i) rough registration using a centerline representation and (ii) fine registration using the mesh representation.

The initial centerline is computed by equidistantly slicing the mesh parallel to a plane defined by the open contour at the bottom of the impression (Fig. 1).

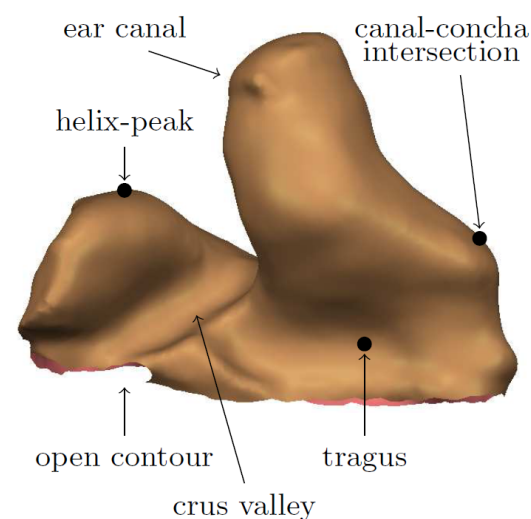

(a) Frontal view

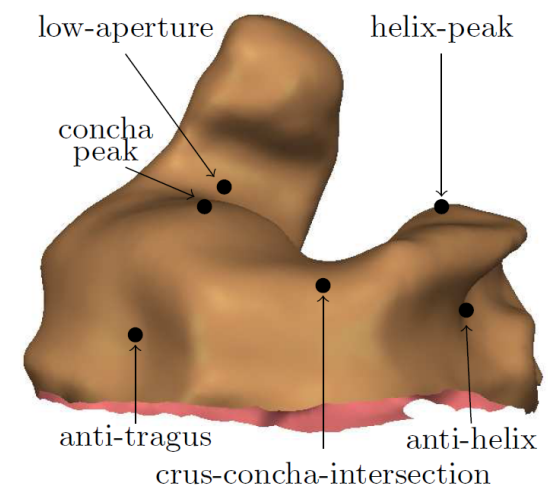

(b) Backward view

Fig. 1. 3-D mesh representation of an ear impression (ear negative) consisting of the external ear and the ear canal. Some of the later considered features are annotated. 
For each slice the center of mass is computed resulting in an ordered set of points $\boldsymbol{l}_{1}, \ldots, \boldsymbol{l}_{N}$. Afterwards the centerline is refined and improved by applying internal and external forces as defined below

$$
\begin{aligned}
E_{\mathrm{int}, i} & =\boldsymbol{l}_{i-1}+\boldsymbol{l}_{i+1}-2 \boldsymbol{l}_{i} \\
E_{\mathrm{ext}, i} & =\frac{1}{N} \sum_{r=1}^{N} \frac{\boldsymbol{x}_{r, i}}{\left|\boldsymbol{x}_{r, i}\right|} \\
\boldsymbol{l}_{i}^{\prime} & =\boldsymbol{l}_{i}+\alpha E_{\mathrm{int}, i}+\beta E_{\mathrm{ext}, i}
\end{aligned}
$$

In eqs. (1) to (3), $\boldsymbol{x}_{r, i}$ denotes the intersection of a random ray $\boldsymbol{r}$ with the mesh emitted from the centerline point $\boldsymbol{l}_{i}$, and $N$ is the number of rays. The final update rule of a centerline point $\boldsymbol{l}_{i}$ is a weighted combination of the internal and external force, where $\alpha=0.04$ and $\beta=1.0$. The centerline points are updated according to eq. (3) until convergence. An example for the initial and final centerline is given in Fig. 2. A critical step of the ICP is the matching of point pairs, which can be computational expensive. We can use the fact that the centerlines are ordered from top to bottom to employ an efficient point matching technique. We shift the centerlines along each other. In each shift step the overlapping parts are extracted. The point matching is then reduced to matching the index $i$. For every step, we compute the ICP (point-to-point error metric) and store the transformation matrix along with the registration error. To solve the ICP, we utilize the SVD-based strategy proposed by Arun et al. [7]. For the fine registration an ICP with the point-to-plane error metric is used. It utilizes the surface normals as additional information and, therefore, allows that smooth or planar areas of the meshes slide over each other easily. Here, no closed-form solutions are available. Thus, we linearize the problem using the assumption that incremental rotations are small, which is valid due to our centerline registration.

\subsection{Identification of a Representative Set of Ear Impressions}

To identify a representative set of ear impressions, we aligned a sample set of 473 ear impressions with each other and stored the averaged squared error of each

Fig. 2. Centerline of an ear impression. The initial centerline (jagged) is colored in red and the refined smooth centerline in black. The initial centerline is biased by the concha area (left part of the impression), while the corrected centerline follows the canal.

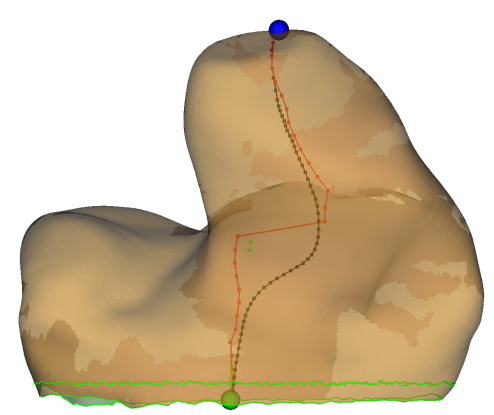


alignment along with its transformation matrix $\mathbf{T}$. This information was given as input into an agglomerative hierarchical clustering (AHC) algorithm. AHC was used, because no evidence for a certain number of clusters was available. We employed the complete-linkage criterion to identify the closest clusters and analyzed the development of the dissimilarity measure along with scatter criteria to identify the most natural clustering. The cluster centers collectively define our representative set of ear impressions $\mathcal{S}_{\text {Rep }}=\left\{S_{1}, \ldots, S_{n}\right\}$. Finally, each member of $\mathcal{S}_{\text {Rep }}$ was annotated by an expert designer.

\subsection{Feature Projection}

To detect the features on a so far unknown ear impression $S_{\text {new }}$ it is registered with each surface in $\mathcal{S}_{\text {Rep }}$. In the following only surface $S_{\text {ref }} \in \mathcal{S}_{\text {Rep }}$ with the smallest registration error is considered. The features $F_{\text {ref }}$ of $S_{\text {ref }}$ are transformed using $\mathbf{T}_{\text {ref }}$ resulting in a new feature set $F_{\text {ref }}^{\prime}$. Since $F_{\text {ref }}^{\prime}$ typically will not end up directly on the surface $S_{\text {new }}$ a final projection is necessary. Currently, we employ only simple projection algorithms. In case of feature points or point sets, the projection is achieved by projecting the given point on the closest point of $S_{\text {new }}$. In case of feature planes, no projection is necessary.

\section{Results}

The clustering of a set of 473 ear impressions resulted in a representative set of 10 ear impressions. Major distinctive properties of the clusters are thickness of the ear canal, direction of the ear canal, thickness and length of the helix and the shape of the anti-tragus concavity (Fig. 1).

For the evaluation of the feature detection we restricted ourselves to the feature points listed in Tab. 1. We compared our CBD scheme with a surfaceanalysis-based (SBD) one developed by Baloch et al. [6]. The results presented in Tab. 1 are based on the evaluation of 117 expert annotated ear impressions. For each feature point the accuracy (Euclidean distance to annotated feature), the detection rate and the tolerance rate is calculated. The latter is defined as not exceeding a certain threshold $\theta$. In agreement with the design experts, $\theta$ was set to $3 \mathrm{~mm}$. The results clearly indicate that the CBD is superior. On average it is $1 \mathrm{~mm}(\approx 32 \%)$ closer to the target and offers a smaller standard deviation compared to the SBD. The achieved detection rates are both very good: $98.8 \%$ for SBD and $100 \%$ for CBD, respectively. As expected from the numbers given in Tab. 1, the tolerance rates differ strongly. The SBD obtained a tolerable result in $62.3 \%$ of the cases, while the CBD was good in $71.0 \%$ of the cases.

\section{Discussion}

A novel clustering-based feature detection scheme specifically adapted to ear impressions was presented. The scheme involves the identification of a representative set of ear impressions and the development of a robust alignment procedure for ear impressions. The feature detection is carried out by registering a 
Table 1. Comparison of SBD and CBD. $\mu$ denotes the mean distance in $\mathrm{mm}$ and $\sigma$ the standard deviation.

\begin{tabular}{lllll}
\hline Feature point & $\mu_{\mathrm{SBD}}$ & $\pm \sigma_{\mathrm{SBD}}$ & $\mu_{\mathrm{CBD}}$ & $\pm \sigma_{\mathrm{CBD}}$ \\
\hline tragus & 2.91 & 2.53 & 1.94 & 1.18 \\
anti-tragus & 3.40 & 3.18 & 2.38 & 2.02 \\
anti-helix & 4.63 & 3.86 & 3.12 & 3.13 \\
helix-peak & 3.62 & 6.42 & 3.14 & 1.91 \\
concha-peak & 3.47 & 3.69 & 2.91 & 2.77 \\
low-aperture & 2.89 & 1.72 & 2.02 & 0.97 \\
crus-concha-intersection & 4.61 & 5.75 & 3.25 & 1.68 \\
canal-concha-intersection & 4.42 & 5.14 & 2.52 & 3.03 \\
canal-crus-intersection & 4.64 & 6.66 & 2.11 & 1.13 \\
\hline Overall & 3.84 & 4.32 & 2.59 & 1.98 \\
\hline
\end{tabular}

new impression with the impressions of the representative set. Followed by a projection of the features taken from the impression with the best registration result. We could show that our scheme is superior compared to an approach that analyzes the surface for peaks, concavities, ridges and bumps. On average an improvement of $1 \mathrm{~mm}$ could be achieved. Due to the template based approach, the detection rate is $100 \%$. The tolerance rate, defined as detecting a feature in a certain area around the labeled feature, could be improved about $9 \%$. Furthermore, the standard deviation of the detection error is greatly reduced, which corresponds to a more robust detection of the features.

So far, the presented results are preliminary and restricted to feature points. The next steps in our work are extending the implementation to feature planes and feature areas and better feature projection algorithms.

\section{References}

1. Sickel K, et al. Semi-automatic manufacturing of customized hearing aids using a feature driven rule-based framework. Proc VMV. 2009; p. 305-12.

2. Paulsen RR, et al. Using a shape model in the design of hearing aids. Proc SPIE. 2004; p. 1304-11.

3. Paulsen RR, et al. Building and testing a statistical shape model of the human ear canal. Proc MICCAI. 2002; p. 373-80.

4. Zouhar A, et al. Anatomically-aware, automatic, and fast registration of 3D ear impression models. Proc 3DPVT. 2006; p. 240-7.

5. Unal GB, et al. Customized design of hearing aids using statistical shape learning. Proc MICCAI. 2008; p. 518-26.

6. Baloch S, et al. Automatic detection of anatomical features on 3D ear impressions for canonical representation. Proc MICCAI. 2010; p. 555-62.

7. Arun KS, Huang TS, Blostein SD. Least-squares fitting of two 3-D point sets. IEEE Trans Pattern Anal Mach Intell. 1987;9(5):698-700. 